\title{
Binding without Identity: Towards a unified semantics for bound and exempt anaphors ${ }^{1}$
}

\author{
Eric Reuland ${ }^{1} \&$ Yoad Winter ${ }^{1}$ \\ ${ }^{1}$ Utrecht institute of Linguistics OTS, Janskerkhof 13, \\ 3512 BL Utrecht, The Netherlands \\ y.winter, e.reuland@uu.nl
}

\begin{abstract}
Expressions such as English himself are interpreted as locally bound anaphors in certain syntactic environments and are exempt from the binding conditions in others. This article provides a unified semantics for himself in both of these uses. Their difference is reduced to the interaction with the syntactic environment. The semantics is based on an extension of the treatment of pronominals in variable-free semantics. The adoption of variable free semantics is inspired by the existence of proxy-readings, which motivate an analysis based on Skolem functions. It is explained why certain anaphor types allow proxy-readings whereas others do not.
\end{abstract}

Keywords: Anaphors, exemption, proxy-readings, Skolem functions, variablefree semantics.

\section{Introduction}

One of the intriguing properties of the English anaphoric system is that members of one and the same class of elements - himself, and the other members of its paradigm must be locally bound (they are subject to condition A of the binding theory) in one set of environments, and is exempt from this local binding requirement in other environments. In such environments the antecedent need not be local. In certain cases a linguistic antecedent may even be absent and a 'logophoric' interpretation obtains. This contrast has been discussed, among others, in Pollard and Sag (1992), Reinhart and Reuland (1991, 1993, henceforth R\&R), and Reuland (2001, 2005a, 2008). A typical set of environments where the contrast shows up in given in (1) and (2):

(1) * Alice expected [the king to invite herself for a drink]

(2) a. Alice expected [the king to invite [the Rabbit and herself] for a drink]

b. Alice expected [the king to invite [no one but herself] for a drink]

(1) illustrates the canonical case of a condition A violation. Herself is an anaphor and must be bound in its local domain, roughly the minimal clause containing it (see Chomsky 1981 for a precise statement of the canonical binding theory). Alice is the

\footnotetext{
${ }^{1}$ The names of the authors appear in alphabetical order. The authors would like to thank Jakub Dotlačil and Anna Volkova for their help in providing the Czech and Russian facts.
} 
only potential antecedent of herself, but is outside the latter's binding domain. Hence, (1) is ill-formed. However, in (2) Alice is even farther away from the anaphor than in (1), yet here Alice can serve as an antecedent for herself, and these sentences are wellformed. This is problematic for the canonical binding theory, not only technically, but also conceptually. As R\&R show, these and other facts - for instance, the differences in distribution between simplex anaphors (henceforth SE-anaphors) and complex anaphors (SELF-anaphors) like Dutch zich and zichzelf and their cognates in other languages - follow if conditions $\mathrm{A}$ and $\mathrm{B}$ are essentially seen as conditions on predicates and stated as follows:

(3) Conditions:
A: A reflexive-marked syntactic predicate is reflexive
B: A reflexive semantic predicate is reflexive-marked

These conditions are based on the following definitions (from $R \& R$ ):

\section{Definitions:}

a. The syntactic predicate of (a head) $\mathrm{P}$ is $\mathrm{P}$, all its syntactic arguments and an external argument of $\mathrm{P}$ (subject)

The syntactic arguments of $\mathrm{P}$ are the projections assigned $\Theta$-role or Case by $\mathrm{P}^{2}$

b. The semantic predicate of $\mathrm{P}$ is $\mathrm{P}$ and all its arguments at the relevant semantic level

c. A predicate is reflexive iff two of its arguments are bound by the same $\lambda$-operator ${ }^{3}$

d. A predicate (of $\mathrm{P}$ ) is reflexive-marked iff either $\mathrm{P}$ is lexically reflexive or one of P's arguments is a SELFanaphor

For the moment we will focus on condition A, and the definitions in (4a, c, and d). It is easily seen that in (1) herself is a syntactic argument of the predicate formed of invite. Therefore, it reflexive-marks it. Condition A, then, requires the predicate to be reflexive. This requirement cannot be met due to a feature mismatch between the king and herself, hence the sentence is ruled out. In (2a,b) herself is not a syntactic argument of invite. Rather, it is properly contained in one (the Rabbit and herself and no one but herself, respectively). Consequently, condition A does not apply, and the predicate is not required to be reflexive. Here the anaphor is exempt, to use Pollard and Sag's term. Hence, no violation of condition A ensues. Where syntactic principles do not enforce an interpretation as a reflexivizer, its eventual interpretation will be determined by general semantic and discourse principles. As is shown extensively by Pollard and Sag himself/herself may end up being bound by a higher c-commanding

\footnotetext{
2 The reference to P includes P's extended projection in the sense of Grimshaw 1991.

${ }^{3}$ The original definition is stated in terms of coindexing. Being bound by the same operator follows the definition of binding in Reinhart (2006). Note that this is not strictly speaking compatible with the variable free approach to be adopted in the body of this article, but for the present purpose this can be ignored.
} 
antecedent, and, if none is available by a suitably prominent discourse entity, and receive a logophoric interpretation.

Summarizing, condition A expresses that the SELF- anaphor enforces reflexivity of the predicate in (1), but not in (2). The questions we need to address concern the way in which condition A is syntactically implemented, and how this affects the semantic interpretation.

\section{The syntax of reflexive-marking}

In a parsimonious theory of grammar, locality of binding should follow from the same general principles that give rise to locality in other domains. Ideally, the grammar should contain no statements specific to binding, except for a definition of binding itself. Similarly, also the interpretation of anaphors in various syntactic contexts should be determined by the same semantic principles applying in a uniform way. That is, also the semantics of anaphoric expressions should be as general as possible.

Reinhart and Reuland (1993) do not discuss a specific syntactic mechanism for reflexive-marking. Reinhart and Reuland (1991), however, suggested that the mechanism involves covert syntactic movement, with SELF moving onto the predicate head by head-movement. In line with earlier approaches such as Helke (1973), we assume that himself is syntactically complex, with SELF being an $\mathrm{N}$ projecting an NP, and him occupying a position in the left periphery as in (5a), where $\mathrm{F}$ is a functional projection, which we can assume to be Person. ${ }^{4}$ When inserted in the proper configuration SELF can be attracted by the head of the predicate, and adjoin to the latter, as in $(5 b)$, which is transparently reflected in nominalizations such as selfhatred or self-admiration.

$$
\begin{array}{ll}
\text { a. } & {\left[_{\mathrm{FP}} \operatorname{him}\left[{ }_{\mathrm{NP}} \mathrm{SELF}\right]\right]} \\
\text { b. } & \mathrm{DP}\left[_{\mathrm{VP}}\left[{ }_{\mathrm{V}} \mathrm{SELF} V\right]\left[_{\mathrm{DP}} \operatorname{him}\left[_{\mathrm{NP}}(\mathrm{SELF})\right]\right]\right]
\end{array}
$$

This idea is elaborated in Reuland (2001, 2005a, and 2008). It is easy to see that the syntactic conditions on exemption follow without further stipulation if the relation between SELF and the predicate is indeed covert syntactic movement. If so, it is sensitive to standard island conditions on movement, specifically head-movement. In (2a) moving SELF onto invite would violate the coordinate structure constraint, in (2) the adjunct island constraint (independently of whether these constraints can be reduced to more fundamental principles of grammar). Hence SELF cannot move onto the predicate in $(2 \mathrm{a}, \mathrm{b})$ and require it to be reflexive, which explains the exemption. ${ }^{5}$

${ }^{4} \mathrm{R} \& \mathrm{R}$ assume that him is in the D-position. For reasons discussed in Reuland (under contract) its position is in a functional projection below $\mathrm{D}$, but for present purposes this issue can be put aside.

${ }^{5}$ It would lead us beyond the scope of the present article to go over all the cases of exemption. See R\&R 1993 for detailed discussion. The reduction of reflexive-marking to SELFmovement has a range of non-trivial empirical consequences, which we cannot possibly go into here. They are discussed in detail in Reuland (under contract). 
A crucial claim of this approach is that English has only one expression himself. And indeed, this is the most parsimonious way to derive the complementarity of exempt and bound uses of himself. However this claim naturally leads to the question of its semantics. Can we find a unified semantics of himself covering both its bound and its exempt uses? The matter is discussed in R\&R (1991), and subsequently, in Reuland (2005a, 2008). The idea pursued there is that SELF is a predicate expressing identity. When adjoined to the predicate head SELF's extension is intersected with the extension of the predicate head, which intuitively conveys the intended meaning. It is less clear what happens in exempt positions. Since SELF is an identity predicate, one of its arguments will be the pronominal. The other argument will have to pick up its value from the context. However, it is not trivial to express the semantics in a compositional way. In the next section we will present a compositional semantics for himself. As we will show, this semantics is extendable to different types of reflexive markers. Many languages, for instance use body-part reflexives. As we will see, our approach naturally applies to such elements.

The basis for our semantics, however, is provided by the solution to another puzzle, which we will introduce first.

\section{Binding and proxies}

Our treatment of the semantics of himself is inspired by one of the well-known properties of reflexive pronouns: their ability to have "proxy readings". This is illustrated in (6) (Jackendoff 1992):

(Upon a visit in a wax museum:) All of a sudden Ringo started undressing himself.

Himself in (6) can refer to the "real" Ringo, but also to a statue of the Ringo denoted by the subject. As Jackendoff argues, the availability of proxy interpretations of reflexives (6) must be related to a general property of language: the ability to refer to various "proxies" of an individual concept. In that respect, the reflexive in (6) is not different from non-anaphoric NPs, which can also refer to "non-canonical" proxies (cf. Ringo/the man is made of stone, whereas Yoko/the woman is made of wax) (see also Safir 2004 for pertinent discussion).

Jackendoff has argued that there is an asymmetry between NP's and anaphors in their ability to carry a proxy reading, and claims that in (7) we cannot have an interpretation where Ringo is the proxy and himself the person.

\section{(7) Ringo fell on top of himself}

However, it is not at all difficult to create contexts where such an interpretation is easily accessible. Consider a play where some actor plays a younger Ringo, and Ringo plays an older fan. It is no problem to interpret the sentence Ringo stumbled and fell on top of himself as true when the actor stumbled and fell on top of the real Ringo. Thus, the availability of proxy-readings represents a general property of 
expressions for individual concepts. Hence the following generalization is expected to hold:

\section{Generalization: The range of available proxies for a bound pronoun} is the same range of proxies as for its antecedent.

Thus, while strict identity between the referents of a pronoun and its antecedent is not mandatory even under binding, identity of the candidate proxy referents for the two expressions is mandatory. This generalization reflects the following observation: also non-reflexive bound pronouns allow a proxy interpretation. For instance:

(9) All of a sudden, every pop icon started taking off the shirt he was wearing.

In the wax-museum context of (6), sentence (9) has a bound reading where the pop icons took the shirts off their respective statues.

This leads to the question of the proper semantics of pronouns. In what one may call the standard analysis of pronouns and anaphors, as summarized in for instance Heim and Kratzer (1998), pronouns and anaphors are essentially variables. Their interpretation is given on the basis of assignments functions.

The fact that pronouns have proxy readings does not come naturally in the standard analysis or pronouns as variables. However, we will show that it is naturally accommodated in an extension of Jacobson's (1999) variable free semantics. Her approach to pronouns dispenses with assignment functions, and also with indices. Pronominals are interpreted as the identity function on entities. It is this conception that provides the basis for generalizing over the bound and exempt uses of himself.

To capture the availability of proxy-readings as in sentence (9) we propose the following natural modification in Jacobson's use of functions. Non-reflexive pronouns like he, instead of simply denoting the identity function on entities, as in Jacobson 1999, denote a Skolem function: a function from entities to entities that takes a relation as a parameter. The formal definition is given in (10):

(10) A function $f$ of type (ee) with a relational parameter $R$ is a Skolem function if for every entity $x: R\left(x, f_{R}(x)\right)$ holds.

We propose that the context provides a proxy relation $(\mathrm{PR})$, describing the possible proxies $\lambda y . P R(x, y)$ of any entity $x$ referred to. This parameter determines the range for each possible entity argument of the Skolem function. We stipulate that any proxy relation must be reflexive. This guarantees availability of the standard interpretation, also in cases like (6) and (9), where the referents for the pronoun and its antecedent entity are strictly identical. This happens because when the relation $R$ is reflexive, one of the Skolem functions $f_{R}$ is the identity function. Thus, our account generalizes Jacobson's use of functions from entities to entities. Sentence (9) is now analyzed as $\left(9^{\prime}\right)$ :

$$
\forall x\left[\text { pop_icon }(x) \rightarrow \text { take off }\left(x, \text { the shirt_ } f_{P R}(x) \text { _ was wearing }\right)\right]
$$


Thus, for every pop icon $x$, the Skolem function $f_{P R}$ picks up one of $x$ 's proxies in the set $\lambda y . P R(x, y)$, possibly $x$ itself. Deriving this analysis is straightforward within Jacobson's framework.

\section{Binding of SELF-anaphors}

What do these considerations imply for reflexive pronouns, in particular SELFanaphors? As indicated in (5), we decompose the anaphor himself into a pronoun him, and self. Since pronominals need not be bound, the relative binding requirement of himself must reside in the self-part. We treat English self (as its cognates in other languages) as a relational noun, denoting a relation between entities and their proxies (with the identity relation as the limiting case). This requirement amounts to assuming that self denotes a reflexive relation: an entity $x$ can have more than one "self" in addition to $x$. In the decompositional semantics of herself, self replaces the contextual proxy-relation of the bare pronoun her.

A noun phrase like Ringo's better self is not substantially different from any other NP with a relational noun (e.g. Ringo's better parent), where the former NP may refer to one of Ringo's "better" proxies in the context of utterance. As noted in section 2, self can incorporate (Van Geenhoven and McNally 2005) with nouns and nominalized transitive verbs. In this it is similar to other relational nouns. For instance:

(11) a. $\quad$ self-hater denotes the predicate $\lambda x$.hate $(x, \uparrow$ self $(x))$

( $x$ is a self-hater if $x$ hates the property (indicated by the $\uparrow$ operator) coupled with $x$ 's proxies)

b. parent-hater denotes the predicate $\lambda x$.hate $(x, \uparrow \operatorname{parent}(x))$

( $x$ is a parent-hater if $x$ hates the property coupled with $x$ 's parents)

The only substantial difference we assume between self and other relational nouns is a syntactic one. The noun self is able to combine with Skolem functions denoted by non-reflexive pronouns independently of genitive case (viz. "?his self/himself vs. his parent/*him parent). There are two ways in which this can happen:

i. The unmarked option - the noun self composes with the Skolem function denoted by the pronoun through the binding mechanism. The noun self covertly incorporates into the transitive predicate (as happens overtly in self-hater) and contributes a proxy relation to the non-reflexive pronoun through Jacobson's $\mathrm{Z}$ function in its "proxied" version:

$$
Z^{P R}=\lambda R \cdot \lambda f \cdot \lambda x \cdot R\left(x, f_{P R}(x)\right)
$$

In this version of the $\mathrm{Z}$ function, it provides the Skolem function $f$ with its parameter. The denotation of a VP like undress himself in (6) is obtained using the structure selfundress him, analyzed as in (13): 


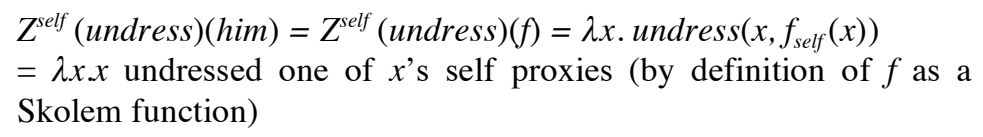

ii. A marked option - the noun self composes with the Skolem function directly. We assume that this marked option is only available in exempt positions, when the incorporation with the predicate is syntactically blocked, as discussed in section 2 . e.g. Max boasted that the queen invited [Lucie and himself] for a drink. When formation of self- $\mathrm{V}$ is syntactically disallowed (as for instance by the the Coordinate Structure Constraint), direct composition with the Skolem function leads to the analysis in (14):

(14) himself $=f_{\text {self }}=$ a function mapping every entity $x$ to one of its proxies in $\operatorname{self}(x)$

Unlike what happens in the unmarked option, now there is no binding that is made necessary by self's composition. As a result, the exempt reading of himself allows it to be interpreted as either bound or free, similarly to the non-reflexive pronoun him.

Hence, self has the same semantics in both cases. The difference resides in how the instruction associated with its semantics is applied. This in turn is determined solely by the syntactic context. The crucial advantage of Jacobson's approach is that it makes available an argument for self that has the proper type in both the bound and the exempt case.

Remains the question of why the option with self-movement is the unmarked case. In Reuland $(2001,2005 a)$ it is argued that the simplest reason resides in a general economy principle,to the effect that encoding binding dependencies in the syntax is cheapest, hence preferred. For current purposes this suffices, see Reuland (under contract) for more extensive discussion.

\section{Simplex anaphors and proxy-interpretation}

Dutch (like the Scandinavian languages) has two anaphors, a SE-anaphor zich, and a SELF-anaphor zichzelf. Zich is an anaphor in the sense that it must be bound (although as its locality condition is less strict, see Reuland 2005b for discussion). R\&R 1993 analyze it as a pronominal that is under-specified. It is not specified for gender and number, but only for the feature $3^{\text {rd }}$ person. Given this, one would expect that it allows proxy-readings like any pronominal. However, there is a clear contrast between the following sentences:

(15) a. Ringo begon zich te wassen.

"Ringo started to wash" no proxy reading

b. Ringo begon zichzelf te wassen.

"Ringo started to wash himself" proxy reading possible 
That is, again in the situation of the wax museum, suppose Ringo finds his statue dirty and wants to do something about it, we can say (15b), but not (15a). What is the source of the difference between the pronouns zich and zichzelf? As in the case of exemption discussed above we would like to find the answer in the syntactic environment, keeping the semantics uniform.

We will relate this to the lexical status of reflexives, coming back to the definition in (1d). A predicate can be reflexive-marked either extrinsically (by a SELF-anaphor), or lexically. Reinhart (2002) and Reinhart and Siloni (2005) present a theory of operations on argument structure (for details we refer to the works cited). One of the options these works allow is a lexical operation, reducing the internal argument and bundling its thematic role with the role of the external argument, as in (16):

(16) Internal Reduction/Bundling $\mathrm{R}_{\mathrm{s}}$ :

$$
\begin{array}{ll}
\text { a. } & \mathrm{V}_{\mathrm{acc}}\left(\theta_{1}, \theta_{2}\right) \rightarrow \mathrm{R}_{\mathrm{s}}(\mathrm{V})\left(\theta_{1,2}\right)\left(\text { where } \theta_{1,2}\right. \text { stands for the } \\
\text { bundling of } \left.\theta_{1} \text { and } \theta_{2}\right) \\
\text { b. } & \mathrm{R}_{\mathrm{s}}(\mathrm{V})\left(\theta_{1,2}\right) \leftarrow \theta_{1,2}(\lambda \mathrm{x}(\mathrm{V}(\mathrm{x}, \mathrm{x})))
\end{array}
$$

The operation is available for a subclass of transitive/accusative assigning verbs, including verbs such as wash, shave, etc. Thus, English wash has two related entries, one intransitive and inherently reflexive in John washed, the other transitive in John washed Mary/John washed himself. The same contrast applies to Dutch. One of the cross-linguistic variables is how the reduction operation affects accusative Case. In languages of the English type the reduction also affects accusative Case. The reduced entry is not an accusative Case assigner, hence no further operation is necessary. In Dutch, with a somewhat richer Case system than English, structural accusative is preserved under reduction. Hence an element has to be inserted checking this Case. Crucially, the element to be inserted should not be interpreted as an independent syntactic argument. For reasons discussed in Reuland (2001) zich's feature deficiency allows it to form one syntactic object with the subject, technically a chain.

Thus, our analysis of the chain in the intransitive usage of waste ("washed") in (15a) yields the interpretation in (17):

$$
\|[\text { Jan,zich }] \|=f_{P R}(\text { jan })=\text { one of Jan's proxies. }
$$

That is, the proxy-function interpreting zich "skips" the predicate waste, and applies directly to the subject. The resulting interpretation is indistinguishable from the "simple" denotation "jan" of the name Jan, given the generalization (8) that any referential NP can be interpreted as any member of the relative set of proxies. Thus, interpreting zich by a proxy-function meshes well with the syntactic structure, without further assumptions being necessary.

By contrast, in (15b), similarly to (6), the reflexive pronoun fills in a separate (object) argument position of a transitive verb (here, the transitive reading of waste). As a result, the analysis of (15b) is similar to the binding with the English sentence Jan washed himself. 
So, zich reflects what one may call strict binding. This tallies with the fact that in intensional contexts zich only allows a de se interpretation. In terms of Chierchia's (1989) discussion, Pavarotti zag zich in de film, maar realiseerde zich niet dat hij het zelf was "Pavarotti saw SE in the movie, but didn't realize it was he hemself" has the air of contradiction, but the result of replacing zich by zichzelf is fine. For completeness sake, note that due to the defective nature of zich, the complex form zichzelf is always bound. For details we refer to Reuland (under contract).

Lexical reflexivization as in Dutch or English is limited to a subclass of transitive verbs. In other languages bundling applies productively. As argued by Reinhart (2002) and Reinhart and Siloni (2005), in such languages bundling applies in the syntax. That is, verbs to be reflexivized project two syntactic arguments. The class of languages of this type includes French, Italian, Czech, and others. Interestingly, bundling in the syntax is compatible with the availability of proxy readings. Russian, which has only restricted lexical reflexivization marked by the $s^{\prime} a$-affix and Czech form a nice minimal pair as illustrated in (18) and (19).
a. nedavno, posetivšij muzej, Ringo pomyls'a (=Ringo, *statue) recently, having visited the museum, Ringo washed-aff
b. nedavno, posetivšij muzej, Ringo pomyl seb'a (=statue, ?Ringo)
recently, having visited the museum, Ringo washed himself
a. Ringo se začal prohlížet (=statue, Ringo)
Ringo started to look at himself
b. Ringo mluvil o svém vzhledu (=statue, Ringo)
Ringo talked about his appearance

This contrast follows if syntactically projected argument positions have the same semantic status as pronouns.

\section{Extending the approach}

Many languages have yet different strategies of reflexivization. In the language sample studied in Schladt (2000) the most frequent reflexivization strategy used socalled body-part (BP) reflexives, as for instance in Basque (20), which uses the expression his head as an anaphor.

(20) a. aitak bere burua hil du father+ERG 3SGPOSS head+NOMDEF kill have+3SG+3SG

The father killed himself

b. bere buruan txapela ipini du 3SGPOSS head+LOCDEF cap+NOM put have+3SG+3SG

He put the cap on his head 
As illustrated in (20b) this expression can still be used in its literal meaning as well. The question is how the reflexivizing use of his head can be understood.

What body-part expressions have in common with self is that they are inherently relational. Just like any self is some individual's self, a body-part belongs to some individual's body. Pursuing the analysis established in section 4, we will claim that the head of the BP is able to combine with Skolem functions denoted by the nonreflexive pronoun in its POSS position (null or overt). If so, the denotation of a VP like $V$ PronBP is obtained using the structure $B P-V$ him, which is analyzed just like undress himself in (6). The relevant interpretation is therefore given in (21):

$$
\begin{aligned}
& \mathrm{Z}^{\mathrm{BP}}(\mathrm{V})(\text { Pron } \mathrm{BP})=\mathrm{Z}^{\mathrm{BP}}(\mathrm{V})(\mathrm{f})=\lambda \mathrm{x} . \mathrm{V}\left(\mathrm{x}, \mathrm{f}_{\mathrm{BP}}(\mathrm{x})\right) \\
& =\lambda \mathrm{x} \cdot \mathrm{x} \text { V-ed one of } \mathrm{x} \text { 's body's proxies (by definition of } \mathrm{f} \text { as a } \\
& \text { Skolem function) }
\end{aligned}
$$

It is an intriguing question to what extent and under what conditions body-part anaphors are subject to similar exemption effects as English SELF-anaphors. This is a matter for further investigation.

\section{Conclusion}

Our extension of variable-free semantics allows us to naturally accommodate proxyreadings. It generalizes over proxy-readings for pronominals and anaphors. It allows us to unify the semantics of bound and exempt anaphors, and it provides a natural extension from SELF-anaphors to body-part reflexives. Finally, it allows us to unify the semantics of zich where it tails a chain to check a residual case with the general semantics or pronouns. It provides us with a principled means to further investigate the cross-linguistic parameters determining the availability of proxy-readings.

\section{References}

Chierchia, Gennaro. 1989. Anaphora and attitudes de se. In Semantics and contextual expression, ed. Renate Bartsch, Johan van Benthem and Peter van Emde Boas. Dordrecht: Foris.

Chomsky, Noam. 1981. Lectures on Government and Binding. Dordrecht: Foris

Heim, Irene and Angelika Kratzer. 1998. Semantics in Generative Grammar. Oxford: Blackwell Publishers Ltd.

Helke, Michael. 1973. On reflexives in English. Linguistics 106, 5-23.

Jackendoff, Ray. 1992. Mme. Tussaud meets the Binding Theory. Natural Language and Linguistic Theory 10: 1-33.

Jacobson, Pauline. 1999. Towards a variable-free semantics. Linguistic and Philosophy 22, 117-184

Van Geenhoven, Veerle and Louise McNally 2005. On the property analysis of opaque complements. Lingua 115. 
Pollard, Carl and Ivan Sag. 1992. Anaphors in English and the scope of the Binding theory. Linguistic Inquiry 23: 261-305

Reinhart, Tanya. 2002. The Theta System - an Overview. Theoretical Linguistics 28(3), 229-290

Reinhart, Tanya. 2006. Interface Strategies: Optimal and Costly Computations. Cambridge, Mass.: MIT Press

Reinhart, Tanya. and Eric Reuland. 1991. Anaphors and Logophors: An Argument Structure Perspective, in Jan Koster and Eric. Reuland (eds.), Long Distance Anaphora, 283-321. Cambridge University Press, Cambridge.

Reinhart, Tanya, and Eric Reuland. 1993. Reflexivity. Linguistic Inquiry 24.4, 657 $-720$

Reinhart, Tanya and Tal Siloni. 2005. The Lexicon-Syntax Parameter: Reflexivization and Other Arity Operations. Linguistic Inquiry, 389 - 436

Reuland, Eric. 2001. Primitives of Binding. Linguistic Inquiry 32.2, 439-492

Reuland, Eric. 2005a. Binding Conditions: How are they Derived? Proceedings of the 12th International Conference on Head-Driven Phrase Structure Grammar, ed. Stephan Müller. Stanford: CSLI.

Reuland, Eric. 2005b. Long Distance Anaphors in Germanic Languages. In The Syntactic Compendium, ed. Martin Everaert and Henk van Riemsdijk. Oxford: Blackwell

Reuland, Eric. 2008. Anaphoric dependencies: How are they encoded? Towards a derivation-based typology. In Reciprocals and Reflexives - Cross-linguistic and theoretical explorations, ed. Ekkehard König \& Volker Gast. Berlin: Mouton de Gruyter, 502-559

Reuland, Eric. Under contract. Anaphora and Language Design. Cambridge, MA:MIT Press

Safir, Ken. 2004. The Syntax of Anaphora. Oxford: Oxford University Press

Schladt, Mathias. 2000: The typology and grammaticalization of reflexives. In Reflexives: Forms and Functions, ed. Zygmunt Frajzyngier and Traci Curl. Amsterdam: Benjamins. 